\title{
A Study on Parameter Estimation for General Aviation Canard Aircraft
}

\author{
Eung Tai Kim* and Kie-Jeong Seong** \\ Flight Control Research Team, Korea Aerospace Research Institute, Deajeon 34133, Korea
}

\section{Yeong-Cheol Kim***}

Agency for Defense Development, Deajeon 34188, Korea

\begin{abstract}
This paper presents the procedures used for estimating the stability and control derivatives of a general aviation canard aircraft from flight data. The maximum likelihood estimation method which accounts for both process and measurement noise was used for the flight data analysis of a four seat canard aircraft, the Firefly. Without relying on the parameter estimation method, several aerodynamic derivatives were obtained by analyzing the steady state flight data. A wind tunnel test, a flight test of a $1 / 4$ scaled remotely controlled model aircraft, and the prediction of aerodynamic coefficients using the USAF Stability and Control Digital Data Compendium (DATCOM), Advanced Aircraft Analysis (AAA), and Computer Fluid Dynamics (CFD) were performed during the development phase of the Firefly and the results were compared with flight determined derivatives of a full scaled flight prototype. A correlation between the results from each method could be used for the design of the canard aircraft as well as for building the aerodynamic database.
\end{abstract}

Key words: canard aircraft, parameter estimation, flight test, stability derivatives

\section{Introduction}

In general, the primary advantage of the canard aircraft is its inherent stall-proof characteristic that can be achieved by the proper design of enabling the canard to stall earlier than the main wing. A typical small canard aircraft is commonly configured with vertical tails mounted at the tips of main wing as winglets. These tip surfaces should be placed back far enough to be able to function as vertical tails. This requires large wing sweepback, resulting in poor Dutch roll characteristic and insufficient directional control power[1].

The Korea Aerospace Research Institute (KARI) has developed a four seat general aviation canard aircraft named the Firefly [2-6]. The main goal of developing the Firefly was to improve lateral-directional flight characteristics. The flight test of the Velocity, which is a typical four seat canard aircraft as shown in Fig. 1, was carried out before the development of the Firefly to investigate the flight characteristics of the canard aircraft [7-9].

The major geometric differences between the Firefly and the Velocity are the location of the vertical tails and the main wing sweepback angle as shown in Fig. 2. The vertical tails of the Velocity are attached at the wing tips, while the vertical tails of the Firefly are moved backward by locating them at the

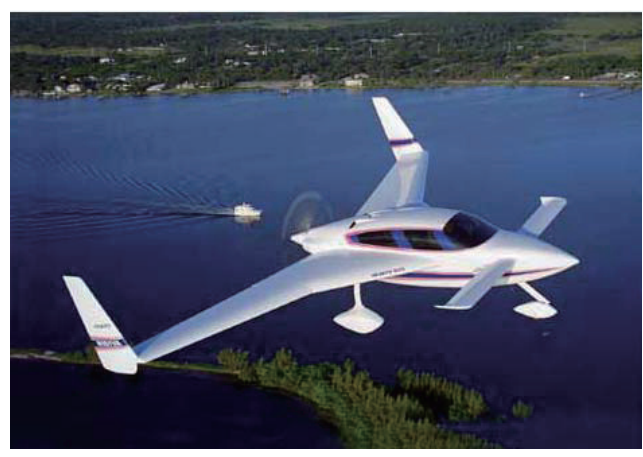

Fig. 1. The Velocity in Flight
This is an Open Access article distributed under the terms of the Creative Commons Attribution Non-Commercial License (http://creativecommons.org/licenses/by$\mathrm{nc} / 3.0 /$ which permits unrestricted non-commercial use, distribution, and reproduction in any medium, provided the original work is properly cited.

\footnotetext{
(c) * Principal Research Engineer, Corresponding Author: eungkim@kari.re.kr ** Principal Research Engineer
} *** Senior Research Engineer 
end of each boom attached to the main wing trailing edge. The main wing leading edge sweepback angle of the Firefly is 18 degrees, which is 5 degrees less than that of the Velocity. The specifications of the Firefly are listed in Table 1.

In the conceptual design phase for the development of the Firefly, the initial configuration was determined from the aerodynamic analysis using DATCOM and AAA. In the preliminary design phase, aerodynamic coefficients were computed from the CFD analysis and measured in the wind tunnel test of a $1 / 4$ scaled model. Prior to the first flight of the full scaled flight prototype, the flight test of a $1 / 4$ scaled remotely controlled model aircraft was conducted to confirm the dynamic flight characteristics and flight safety. Finally, the flight test of a flight prototype was performed and the flight data acquired during the flight test was analyzed.

Test and analysis methodologies used during the development process of aircraft have their own inherent properties. Since limitations can exist on the application of their results, it may not be desirable to use these results directly. Correlations between different methods need to be studied. Furthermore, the Firefly has an unusual configuration of canard with vertical tails on tail booms. Therefore, the aircraft design and analysis software currently available may not have an aerodynamic database appropriate

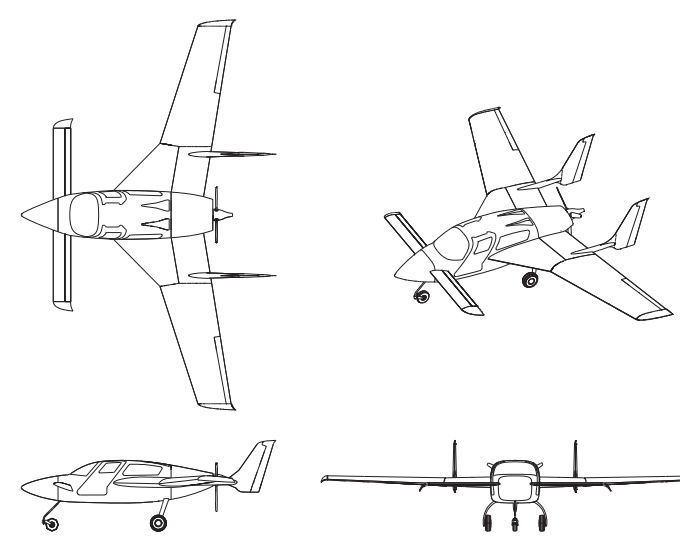

Fig. 2. The Firefly Drawing

Table 1. The Firefly Specification

\begin{tabular}{lr}
\hline Specification & \\
Max. T/O weight, lbs & Value \\
Overall length, $\mathrm{ft}$ & 2700 \\
Overall height, $\mathrm{ft}$ & 21.5 \\
Main wing span, $\mathrm{ft}$ & 7.7 \\
Main wing area, $\mathrm{ft}^{2}$ & 34.2 \\
MAC of main wing, $\mathrm{ft}$ & 120 \\
Canard span, $\mathrm{ft}$ & 4.69 \\
Canard area, $\mathrm{ft}^{2}$ & 15.8 \\
Vertical tail area (total), $\mathrm{ft}^{2}$ & 18.0 \\
CG location, in & 17.2 \\
Performance & $\mathrm{FS} 130$ \\
Minimum speed, kt & Value \\
Cruise speed, $\mathrm{kt}$ & 64 \\
Max. rate of climb, fpm & 167 \\
T/O length, $\mathrm{ft}$ & 1,200 \\
L/D length, $\mathrm{ft}$ & 1,140 \\
Max. range, $\mathrm{nm}$ & 930 \\
& 980 \\
\hline
\end{tabular}

for the configuration of the Firefly.

In this study, various methodologies used in the design of the Firefly for the purpose of predicting flying and handling qualities are presented. Flight test and parameter estimation techniques are discussed. Results from the various methods in the design phase are compared with the estimation results obtained from the flight data of the flight prototype. Flightdetermined derivatives of the Firefly are also compared with those of the Velocity and the Cessna 182.

\section{Prediction of Aerodynamic Derivatives}

In the design phase, several methods have been used to predict the aerodynamic derivatives of the Firefly. These methods are introduced in this section.

A 1/4 scaled model as shown in Fig. 3 was tested in the low speed wind tunnel of KARI [3-4]. Static forces and moment data were measured by a pyramidal type external 6-components strain gauge balance. Most of the tests were performed at the speed of $50 \mathrm{~m} / \mathrm{s}$ and the Reynolds number for this wind tunnel test condition is $1.2 \times 10^{6}$, while the Reynolds number for the flight test is $3.0 \times 10^{6}$ at stall condition and $6.1 \times 10^{6}$ at cruise condition. These differences in the Reynolds number do not noticeably influence the stability and control derivatives, except at the high angle of attack conditions. Only static aerodynamic derivatives were measured in the wind tunnel test.

A 1/4 scaled remotely controlled model aircraft as shown in Fig. 4 was made and instrumented with sensors and data recording device. The maximum speed of this $\mathrm{R} / \mathrm{C}$ aircraft is $48 \mathrm{~m} / \mathrm{sec}$ and the corresponding Reynolds number is almost the same as that for the wind tunnel test condition. The stall speed is $19 \mathrm{~m} / \mathrm{sec}$ and the corresponding Reynolds number is $0.46 \times 10^{6}$, which is regarded as too low. Due to the limitations in the qualities of the sensors and the data recording device that can be installed in the $1 / 4$ scaled model, the accuracy of the flight data is below expectation.

For the CFD analysis, a delta form finite differential

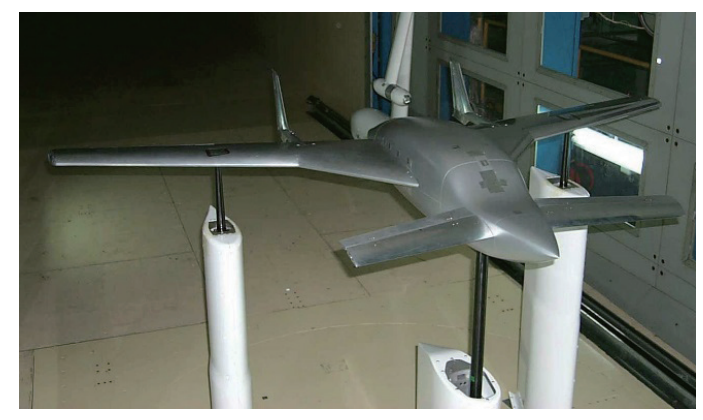

Fig. 3. Wind Tunnel Test of the 1/4 scaled Firelfy model 
equation was derived by applying the Approximate Factorization algorithm to a three-dimensional Euler equation and solved to estimate the aerodynamic characteristics of the Firefly [5]. The grids in the space around the fuselage, canard, and main wing were generated separately and then positioned to overlap each other using the Chimera grid method. The vertical tails were omitted due to the complexity in generating and overlapping the grids, and therefore only the longitudinal aerodynamic characteristics were investigated. The control surfaces were also not modeled due to high complexity and the control derivatives were not computed. Therefore, the output that can be obtained by this CFD analysis for the comparison with flight test data is very limited.

The USAF Stability and Control Digital Data Compendium (DATCOM) was used to calculate the dynamic damping coefficients that cannot be obtained from the wind tunnel test and the CFD analysis. DATCOM is an analytic method of estimating stability coefficients using empirical data of the aircraft [10].

Advanced Aircraft Analysis (AAA) is a preliminary design software for airplanes. AAA is programmed based on the equations and empirical data of ref. [11], some of which are identical or close to those used in DATCOM.

Moments of inertia of all structural parts, subsystems, fuel, pilot, and passengers were calculated and then summed to obtain the moments of inertia of the aircraft.

\section{Flight Test Techniques for Estimation of Aerodynamic Derivatives}

In the flight test of the Firefly, several maneuvers were performed to separately obtain longitudinal and lateraldirectional aerodynamic derivatives. Parameter estimation was performed using dynamic stability and control test data. Each maneuver was performed repeatedly to obtain statistically reliable data.

Since all parameter estimation techniques depend on the

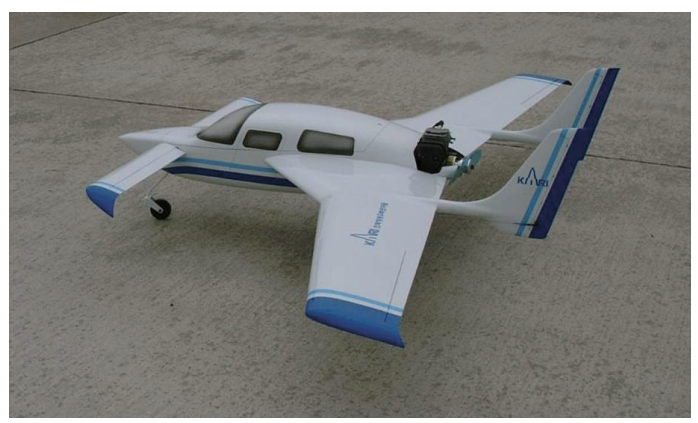

Fig. 4. 1/4 Scaled RC Aircraft control inputs exciting the dynamics of aircraft, determining the proper or optimal control inputs is important in a flight test. This optimal input produces the best response of the aircraft, with the greatest amount of information about the dynamics of the aircraft, to determine the complete set of stability and control derivatives. A variety of input forms have been proposed. However, many of these input forms have not been applied in the flight test for a number of reasons. The most common reason is the inability of the pilot to precisely reproduce a desired but complex input [12]. A short-period longitudinal mode can be excited by a simple pulse or doublet input on the elevator or other longitudinal control surface. For the best excitation, pulse duration should be approximately equal to the natural period of the short-period mode. In this study, modified doublet inputs were applied. The best way to ensure adequate excitation of all lateral-directional modes such as Dutch-roll, roll, and spiral modes is to apply both aileron and rudder inputs during the maneuver. It is certainly possible to excite all modes with aileron input only or with rudder input only; however, these approaches require careful input design and execution. In this study, we adopted a simple rudder doublet input followed by an aileron doublet input.

The commonly used flight test techniques to obtain longitudinal stability data are relatively simple and straightforward. Lift coefficient and pitching moment coefficient can be estimated from a series of steady state flight tests conducted at various C.G. conditions. The trim conditions must be established carefully.

Lateral and directional static stability and controllability could be measured from the flight data by determining the rolling and yawing moments generated by the aileron and rudder, respectively, to keep the aircraft in steady state condition. Unfortunately, lateral static stability and directional static stability cannot be separately measured because they are coupled through sideslip angle, so a technique must be found that would yield lateral and directional static stability measurements simultaneously. One of the maneuvers that satisfy these requirements is a steady-heading sideslip flight or crab test, which can be performed by balancing the forces and moments applied on the aircraft so that it flies at constant sideslip angle while maintaining constant heading. Fig. 5 shows the flight test data of Firefly's crab maneuver.

Roll rate tests are conducted in order to identify rolling characteristics as well as the maximum roll rate. Once the aircraft is stabilized in a 60 degree left bank with zero roll rate, the pilot abruptly moves the stick to the right and holds it until the aircraft is rolled to a 60 degree right bank. The pilot then moves the stick to the neutral position and maintains 
the aircraft at 60 degrees of the right bank angle [13-14], as shown in Fig. 6.

\section{Parameter Estimation}

\subsection{Maximum Likelihood Estimation}

The system under investigation is assumed to be modeled by a set of dynamic equations containing unknown parameters. To determine the values of the unknown
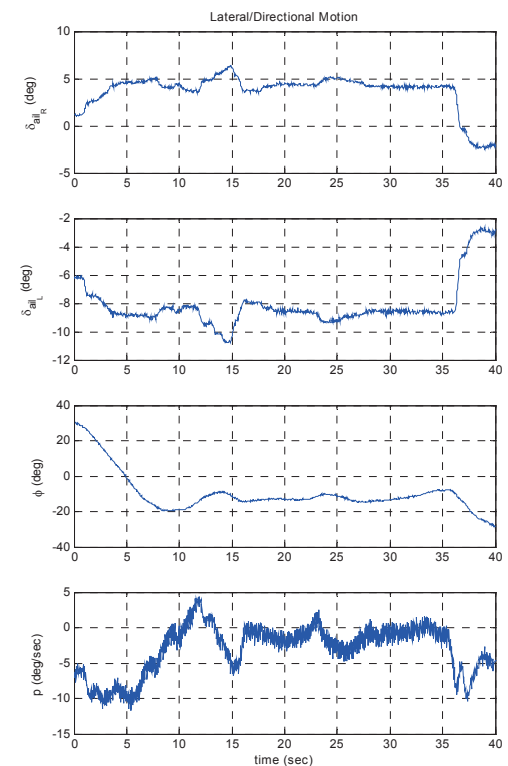

Fig. 5. Crab Flight Test Data
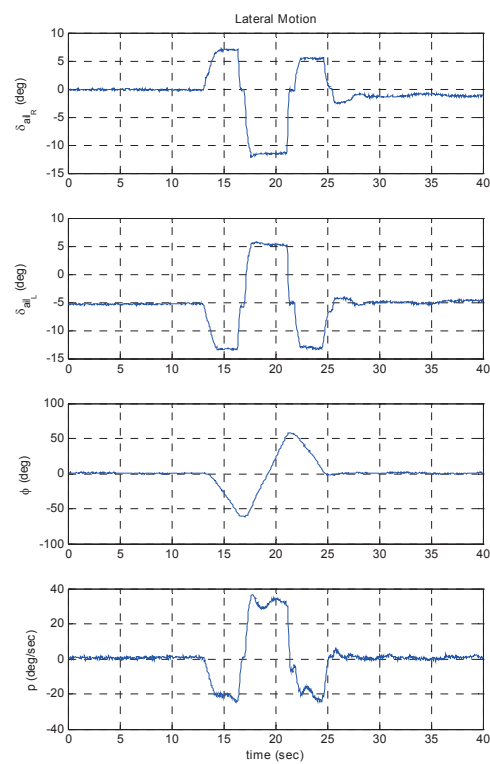

parameters, the system is excited by a suitable input, and the input and actual system responses are measured. The values of the unknown parameters are then inferred based on the requirement that model responses to the given input match the actual system responses.

The Maximum Likelihood Estimation (MLE) method [15-18], which accounts for both process and measurement noise, was used in this research. The algorithm is based on the Kalman filter for the linear state estimation problem, an explicit estimation of covariance of residuals, and the GaussNewton method for parameter update.
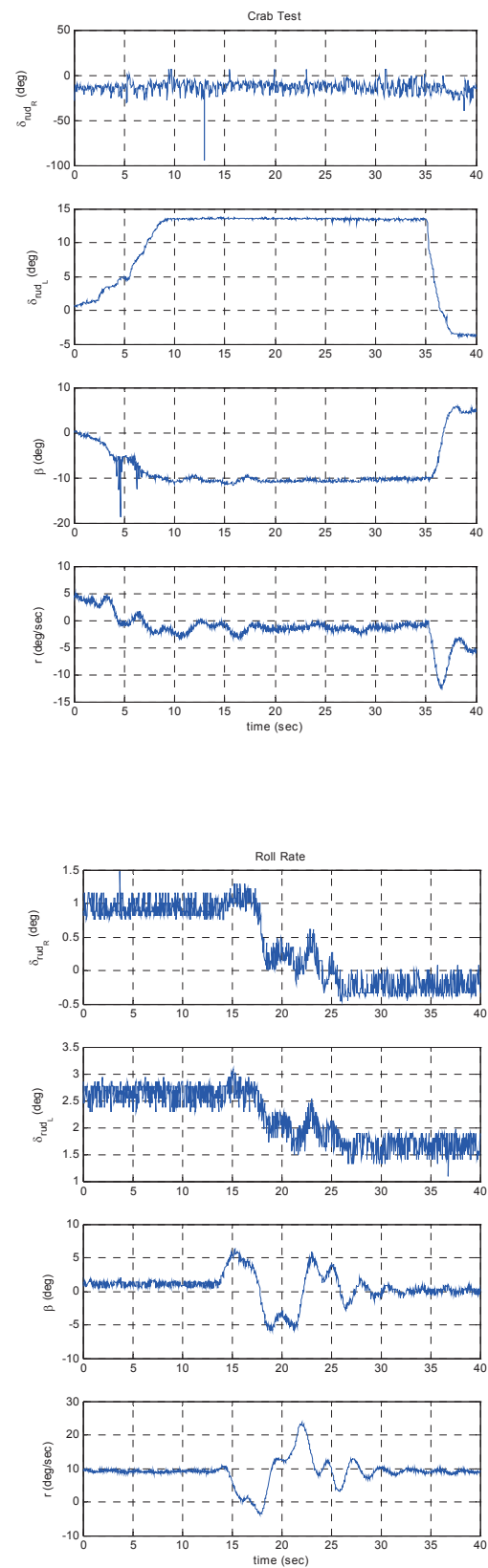

Fig. 6. Roll Rate Flight Test Data 
The dynamic system, of which the parameters are to be estimated, is assumed to be described by the following mathematical model:

$$
\begin{aligned}
\dot{x}(t) & =f[x(t), u(t), \theta]+F w(t) \\
y(t) & =g[x(t), u(t), \theta] \\
z(k) & =y(k)+v(k), k=1, \ldots, N
\end{aligned}
$$

where $x$ is the $(n \times 1)$ state vector, $u$ is the $(p \times 1)$ control input vector, and $y$ is the $(m \times 1)$ observation vector. The process noise, $w(t)$ is assumed to be characterized by a zero-mean Gaussian noise with an identity spectral density. The measurement noise vector, $v(k)$ is assumed to be zero-mean Gaussian with identity covariance.

The maximum likelihood estimates are obtained by minimizing the negative logarithm of the likelihood function:

$$
\begin{aligned}
& \hat{y}(k)=g[\hat{x}(k), u(t), \theta] \\
& \varepsilon(k)=z(k)-\hat{y}(k) \\
& W(k)=E\left\{\varepsilon(k) \varepsilon(k)^{T}\right\} \\
& J(\theta, W)=\frac{1}{2} \sum_{k=1}^{N} \varepsilon(k)^{T} W(k)^{-1} \varepsilon(k)+\frac{1}{2} \sum_{k=1}^{N} 1 n|W(k)|
\end{aligned}
$$

where $W(k)$ is the covariance matrix of residuals.

In order to obtain predicted state variables $\hat{x}(k)$ from the dynamic model with both process noise and measurement noise, the extended Kalman filter, which is an optimal linear state estimator, is used.

In general, minimization of the cost function requires nonlinear programming. It assumes most parts of parameter estimation process and plays major roles in determining efficiency and applicability. Since cost function has a quadratic form, the Newton-Raphson method could be applied to minimize cost function. However, this method is very complex because of the computation of the Hessian. This complexity can be reduced by using the Gauss-Newton method, which uses an approximation to the Hessian.

The Maximum Likelihood Estimator provides a measure of the reliability of each estimated parameter based on the information obtained from each dynamic maneuver. This measure of the reliability is called the Cramer-Rao bound. The Cramer-Rao bound, analogous to the standard deviation, should generally be used as a measure of relative, rather than absolute, accuracy. The bound is obtained from the approximation to the information matrix $H$. The bound for each unknown coefficient is the square root of the corresponding diagonal element of $H^{-1}$; that is, for the $i^{\text {th }}$ unknown parameter, the Cramer-Rao bound is $\sqrt{H_{i, i}^{-1}}$.

\subsection{Parameter Estimation Procedure}

Short period flight test data was used to extract longitudinal stability and control derivatives. The initial values of parameters required at the startup of the estimation algorithm were determined by the AAA results combined with wind tunnel data.

Determining the parameter estimation structure (system model) is important for the estimation. For lateral-directional motion, three different parameter estimation structures are proposed and compared.

i) Case I: Conventional system model

ii) Case II: Case I with one constraint

iii) Case III: Case I with three parameters fixed

Case I structure is based on the linear state-space equations of motion which are derived by applying small perturbation theory to a flight dynamic model. The linear equations of motion decoupled for the longitudinal and lateral-directional motion are shown in Eq. (8) and Eq. (9), respectively.

$$
\begin{aligned}
& {\left[\begin{array}{c}
\Delta \dot{u} \\
\Delta \dot{\alpha} \\
\Delta \dot{q} \\
\Delta \dot{\theta}
\end{array}\right]=\left[\begin{array}{cccc}
X_{u} & X_{\alpha} & 0 & -g \\
Z_{u} & Z_{\alpha} & 1 & 0 \\
\tilde{M}_{u} & \tilde{M}_{\alpha} & \tilde{M}_{q} & 0 \\
0 & 0 & 1 & 0
\end{array}\right]\left[\begin{array}{c}
\Delta u \\
\Delta \alpha \\
\Delta q \\
\Delta \theta
\end{array}\right]+\left[\begin{array}{c}
X_{\delta_{e}} \\
Z_{\delta_{e}} \\
\tilde{M}_{\delta_{e}} \\
0
\end{array}\right] \Delta \delta_{e}} \\
& {\left[\begin{array}{c}
\Delta \dot{\beta} \\
\Delta \dot{p} \\
\Delta \dot{r} \\
\Delta \dot{\phi}
\end{array}\right]=\left[\begin{array}{cccc}
Y_{\beta} & Y_{p} & Y_{r} & \frac{g \cos \theta_{0}}{u_{0}} \\
L_{\beta} & L_{p} & L_{r} & 0 \\
N_{\beta} & N_{p} & N_{r} & 0 \\
0 & 1 & 0 & 0
\end{array}\right]\left[\begin{array}{c}
\Delta \beta \\
\Delta p \\
\Delta r \\
\Delta \phi
\end{array}\right]+\left[\begin{array}{cc}
0 & Y_{\delta_{r}} \\
L_{\delta_{a}} & L_{\delta_{r}} \\
N_{\delta_{a}} & N_{\delta_{r}} \\
0 & 0
\end{array}\right]\left[\begin{array}{l}
\Delta \delta_{a} \\
\Delta \delta_{r}
\end{array}\right]}
\end{aligned}
$$

The Case II parameter estimation structure reduces the number of unknown parameters by introducing the following relation between yawing moment and side force due to rudder deflection.

$$
Y_{\delta_{r}}=\frac{-1}{\operatorname{mass}} \cdot \frac{I_{z z}}{l_{v}} \cdot N_{\delta_{r}}
$$

where $l_{v}$ is the distance from the aircraft C.G. to the vertical tail aerodynamic center and $I_{Z Z}$ is the moment of inertia about $z$-axis.

The result of Case I estimation showed larger scatter and uncertainty level of side force estimates compared with Case II. Therefore, the constraint of the side force derivative using yawing moment derivative could give a better result.

Case III performs parameter estimation by fixing three parameters as constants in the system model. The values of 
$C_{l_{p}}, C_{n_{r}}$, and $C_{n_{\beta}}$ are fixed as the values estimated from Case I and then the remaining derivatives are estimated.

\subsection{Estimation of Static Stability and Control Deriva- tives}

Various parameter estimation methods could be used to extract stability and control derivatives from flight data. These methods introduce statistical techniques based on the minimization of the errors between flight data and simulation results of the estimated model. The most accurate stability and control derivatives could be determined from parameter estimation. However, these estimation methods have limitations since many numbers of parameters are estimated at the same time. It is reasonable to judge that some parameters estimated from steady state flight data would be more accurate and therefore can be used in the verification or analysis of the parameter estimation results [19-21]. The estimation procedures of $C_{L_{\alpha}}$ and $C_{M_{\alpha}}$ from the steady state flight test data are described below.

The neutral point of aircraft is defined as a position about which pitching moment is independent of the angle of attack. For the purpose of calculating the neutral point, the following moment equilibrium equation at the C.G. point can be used:

$$
M_{c g}=-\left(L-L_{\delta_{e}}\right) l_{a}+L_{\delta_{e}} \cdot l_{c}+D \cdot h_{a}-T \cdot h_{e}+M_{a c}=0
$$

where

$$
\begin{aligned}
& l_{a}=x_{a c}-x_{c g} \\
& l_{c}=x_{c g}-x_{\text {canard }} \\
& h_{a}=z_{c g}-z_{a c} \\
& h_{e}=z_{\text {engine }}-z_{c g}
\end{aligned}
$$

In general, the moment effects of thrust and drag are negligible compared with other terms and Eq. (11) may be rewritten as:

$$
C_{M_{a c}}=\left(C_{L_{0}}+C_{L_{\alpha}} \cdot \alpha\right) \frac{l_{a}}{\bar{c}}-C_{L_{\delta e}} \cdot \delta_{e} \frac{l_{c}}{\bar{c}}
$$

If the above equation is differentiated by the angle of attack, the following equation is obtained.

$$
\frac{\partial C_{M_{a c}}}{\partial \alpha}=C_{L_{\alpha}} \frac{l_{a}}{\bar{c}}-C_{L_{\delta e}} \frac{\partial \delta_{e}}{\partial \alpha} \frac{l_{c}}{\bar{c}}
$$

By the definition of the neutral point, $\frac{\partial C_{M_{a c}}}{\partial \alpha}=0$, Eq. (13) could be reduced to:

$$
\frac{\partial \delta_{e}}{\partial \alpha}=\frac{C_{L_{\alpha}}}{C_{L_{\delta e}}} \frac{l_{a}}{l_{c}}
$$

If the C.G. of the aircraft is located at the neutral point, i.e. $l_{a}=0$, then $\frac{\partial \delta_{e}}{\partial \alpha}=0$ becomes zero.

Flight tests were performed at various C.G. locations. The C.G. location was varied from FS 129 in to FS 132 in. The neutral point could be estimated as illustrated in Fig. 7 by extrapolating the straight line approximation to find the center of gravity that makes $\frac{\partial \delta_{e}}{\partial \alpha}=0$. As shown in Fig. 7, the neutral point of the Firefly is located at FS $138 \mathrm{in}$.

The lift coefficient can be calculated from Eq. (15), which is obtained from the force equilibrium equation for trim condition,

$$
C_{L}=\frac{W \cos \alpha-T \sin \alpha}{0.5 \rho V^{2} S}
$$

and the lift coefficient can be written as follows if the lift due to elevator deflection is neglected.

$$
C_{L}=C_{L_{0}}+C_{L_{\alpha}} \alpha
$$

Longitudinal trim flight was performed at various speeds, and the result is illustrated in Fig. 8, from which $C_{l_{0}}$ and $C_{L_{a}}$ are estimated.

Once $C_{L_{\alpha}}$ and the neutral point location are estimated, $C_{M_{\alpha}}$ is calculated by,

$$
C_{M_{\alpha}}=C_{L_{\alpha}} \frac{x_{c g}-x_{n p}}{\bar{c}}
$$

The estimation procedure of lateral-directional derivatives from the steady state flight data are described below.

At a given bank angle, the steady state, straight line lateraldirectional equation of motion can be written as follows:

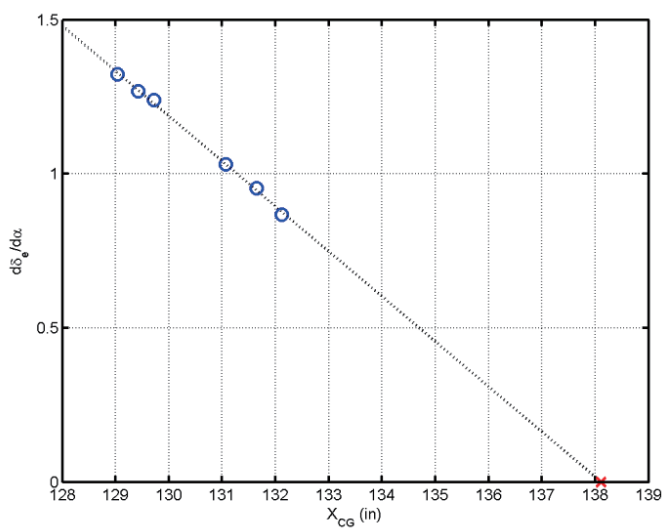

Fig. 7. Neutral Point of the Firefly 


$$
\begin{aligned}
& \left(C_{Y_{\beta}} \cdot \beta+C_{Y_{\delta a}} \cdot \delta_{a}+C_{Y_{\delta r}} \cdot \delta_{r}\right) \cdot \bar{q} s+F_{Y_{T}}=-m g \sin \phi \\
& \left(C_{l_{\beta}} \cdot \beta+C_{l_{\delta a}} \cdot \delta_{a}+C_{l_{\delta r}} \cdot \delta_{r}\right) \cdot \bar{q} s b+L_{T}=0 \\
& \left(C_{n_{\beta}} \cdot \beta+C_{n_{\delta a}} \cdot \delta_{a}+C_{n_{\delta r}} \cdot \delta_{r}\right) \cdot \bar{q} s b+N_{T}=0
\end{aligned}
$$

For the flight conditions with symmetrical thrust, the thrust terms in Eq. (18) may be omitted and Eq. (18) is written as follows:

$$
\begin{aligned}
& C_{Y_{\beta}} \cdot \beta+C_{Y_{\delta a}} \cdot \delta_{a}+C_{Y_{\delta r}} \cdot \delta_{r}=\frac{-m g \sin \phi}{\bar{q} s} \\
& C_{l_{\beta}} \cdot \beta+C_{l_{\delta a}} \cdot \delta_{a}+C_{l_{\delta r}} \cdot \delta_{r}=0 \\
& C_{n_{\beta}} \cdot \beta+C_{n_{\delta a}} \cdot \delta_{a}+C_{n_{\delta r}} \cdot \delta_{r}=0
\end{aligned}
$$

If attitude, velocity, sideslip angle, and control surface deflection data are available from the flight test, stability and control derivatives can be calculated from the simultaneous equations of Eq. (19). Now, we have three equations with nine unknowns. Six further relationships should be introduced in order to solve this problem.

First, the weathercock derivative can be computed from the Dutch roll time history. Dutch roll undamped natural frequency has a relation with lateral and directional derivatives as follows:

$$
\omega_{n_{d}}=\sqrt{\frac{Y_{\beta} N_{r}+U_{0} N_{\beta}-N_{\beta} Y_{r}}{U_{0}}}
$$

The middle term in Eq. (20) usually dominates the lateraldirectional oscillation and the first and second term can be ignored. Dutch roll undamped natural frequency could be approximated as Eq. (21)

$$
\omega_{n_{d}} \approx \sqrt{N_{\beta}} \text { or } C_{n_{\beta}}=\frac{I_{z z}}{\bar{q} s b} \omega_{n_{d}}^{2}
$$

Second, the aileron control derivative $C_{l \delta_{a}}$, and roll

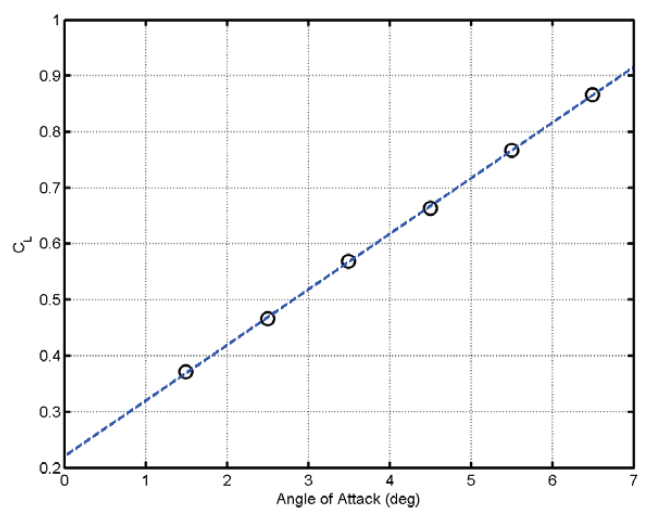

Fig. 8. Lift Curve of the Firefly damping derivative $C_{l_{p}}$ could be computed from the roll rate test. The equilibrium equation for roll motion is as follows:

$$
C_{l_{\delta a}} \cdot \delta a+C_{l_{\beta}} \cdot \beta+C_{l_{p}} \cdot \frac{p b}{2 V}=\frac{I_{x x} \cdot \dot{p}}{\bar{q} S b}
$$

Since the change of sideslip angle during pure roll motion is small, the second term in Eq. (22) related with sideslip angle can be ignored. At steady-state motion, the roll rate $p_{s,}$ is equal to zero and Eq. (22) reduces to Eq. (23).

$$
C_{l_{\delta a}} \cdot \delta a+C_{l_{p}} \cdot \frac{p_{s s} b}{2 V}=0
$$

Third, the following relation between side force and yawing moment induced by rudder deflection is introduced:

$$
C_{n_{\delta_{r}}}=-\frac{l_{v}}{b} C_{Y_{\delta r}}
$$

Fourth, the following relation between side force and rolling moment due to rudder deflection is introduced.

$$
C_{l_{\delta_{r}}}=\frac{z_{v}}{b} C_{Y_{\delta r}}
$$

Fifth, $C_{Y_{\delta \alpha}}$ is assumed to be zero since it is negligibly small for most aircraft. Lastly, for the adverse yaw derivative, $C_{n_{\delta a}}$, which cannot be estimated by the steady state flight

Table 2. MLE Results for Longitudinal Case

\begin{tabular}{ccccc}
\hline \multicolumn{2}{c}{$100 \mathrm{kts}$} & \multicolumn{2}{c}{$120 \mathrm{kts}$} \\
\hline Parameter & Value & CR Bound & Value & \multicolumn{1}{c}{ CR Bound } \\
\hline$C_{D_{0}}$ & 0.0648 & 0.0013 & 0.0501 & 0.0042 \\
\hline$C_{L_{0}}$ & 0.8660 & 0.0024 & 0.5837 & 0.0021 \\
\hline$C_{D_{\alpha}}$ & 0.7528 & 0.0042 & 0.5805 & 0.0073 \\
\hline$C_{L_{\alpha}}$ & 5.4459 & 0.0004 & 5.5669 & 0.0031 \\
\hline$C_{M_{\alpha}}$ & -2.2794 & 0.0009 & -2.3172 & 0.0011 \\
\hline$C_{M_{\alpha}}$ & -0.7932 & 0.0121 & -0.5968 & 0.0412 \\
\hline$C_{M_{q}}$ & -14.684 & 0.0134 & -13.853 & 0.0218 \\
\hline$C_{D_{\delta e}}$ & 0.0000 & 0.0001 & 0.0001 & 0.0000 \\
\hline$C_{L_{\delta e}}$ & 0.2944 & 0.0041 & 0.2543 & 0.0011 \\
\hline$C_{M_{\delta e}}$ & 0.7786 & 0.0091 & 0.7759 & 0.0003 \\
\hline$\zeta_{s p}$ & 0.5768 & - & 0.6273 & - \\
\hline$\omega_{n_{s p}}$ & 4.0197 & - & 4.2020 & - \\
\hline$\omega_{n_{p h}}$ & 0.2731 & - & 0.2171 & - \\
\hline & 0.0425 & - & & -0.0549 \\
\hline
\end{tabular}


maneuver, the output from the maximum likelihood estimation method is used. Now, Eqs. (19), (23), (24), and (25) can be solved for the lateral-directional stability and control derivatives.

\section{Results and Discussion}

The stability and control derivatives obtained from the maximum likelihood estimation method are shown in Table $2 \sim$ Table 4 with uncertainty levels.

The standard deviations of the longitudinal estimates are not presented since flight data for longitudinal motion is insufficient. The Cramer-Rao bounds of the longitudinal derivatives are sufficiently small compared with the magnitude of the estimated parameters, except $C_{D_{\delta e}}$. This implies that estimated parameter values are reliable, except $C_{D_{\delta e}}$, which can be ignored in most cases.

The large differences between the Cramer-Rao bounds and standard deviations of the lateral-directional derivatives shown in Table 4 are possibly caused by the influence of atmospheric turbulence. It is conjectured that the estimated parameters of $C_{n_{\beta}}$ and $C_{l_{p}}$ are accurate since the Cramer-Rao bounds are small and coincide with standard deviations, while the accuracies of $C_{Y_{p}}$ and $C_{n_{\delta a}}$ are quite poor.

Fig. 9 shows a comparison of the flight data and the

Table 3. Maximum Likelihood Estimation Results for Lateral-Directional Case

\begin{tabular}{cccccccccc}
\hline & \multicolumn{3}{c}{ Case I } & \multicolumn{3}{c}{ Case II } & \multicolumn{3}{c}{ Case III } \\
\hline Parameter & $90 \mathrm{kts}$ & $120 \mathrm{kts}$ & $140 \mathrm{kts}$ & $90 \mathrm{kts}$ & $120 \mathrm{kts}$ & $140 \mathrm{kts}$ & $90 \mathrm{kts}$ & $120 \mathrm{kts}$ & $140 \mathrm{kts}$ \\
\hline$C_{Y_{\beta}}$ & -0.7795 & -0.5210 & -0.4240 & -0.9021 & -0.4910 & -0.3901 & -0.8112 & -0.5110 & -0.4340 \\
\hline$C_{l_{\beta}}$ & -0.1012 & -0.0744 & -0.0566 & -0.1311 & -0.0854 & -0.0560 & -0.1134 & -0.0751 & -0.0536 \\
\hline$C_{n_{\beta}}$ & 0.0366 & 0.0351 & 0.0401 & 0.0411 & 0.0384 & 0.0409 & 0.0351 & 0.0363 & 0.0412 \\
\hline$C_{Y_{p}}$ & -0.0013 & -0.0021 & -0.0019 & -0.0011 & -0.0026 & -0.0023 & -0.0014 & -0.0020 & -0.0014 \\
\hline$C_{l_{p}}$ & -0.4600 & -0.4591 & -0.4335 & -0.4820 & -0.4498 & -0.4435 & -0.4701 & -0.4601 & -0.4421 \\
\hline$C_{n_{p}}$ & -0.0506 & -0.0522 & -0.0251 & -0.0406 & -0.0512 & -0.0299 & -0.0510 & -0.0501 & -0.0263 \\
\hline$C_{Y_{r}}$ & 0.1900 & 0.2131 & 0.2324 & 0.2300 & 0.2543 & 0.2514 & 0.1850 & 0.2241 & 0.2321 \\
\hline$C_{l_{r}}$ & 0.1714 & 0.0972 & 0.0651 & 0.1533 & 0.0894 & 0.0591 & 0.1694 & 0.0941 & 0.0655 \\
\hline$C_{n_{r}}$ & -0.0606 & -0.0701 & -0.0652 & -0.0501 & -0.0693 & -0.0612 & -0.0612 & -0.0695 & -0.0659 \\
\hline$C_{l_{\delta a}}$ & 0.1452 & 0.1249 & 0.1102 & 0.1341 & 0.1341 & 0.0902 & 0.1451 & 0.1301 & 0.1201 \\
\hline$C_{n_{\delta a}}$ & -0.0031 & -0.0025 & -0.0014 & -0.0043 & -0.0023 & -0.0018 & -0.0035 & -0.0021 & -0.0011 \\
\hline$C_{Y_{\delta r}}$ & 0.1500 & 0.1491 & 0.1451 & 0.1311 & 0.1451 & 0.1351 & 0.1512 & 0.1521 & 0.1501 \\
\hline$C_{l_{\delta r}}$ & 0.0087 & 0.0105 & 0.0084 & 0.0067 & 0.0113 & 0.0081 & 0.0090 & 0.0112 & 0.0082 \\
\hline$C_{n_{\delta r}}$ & -0.0470 & -0.0410 & -0.0374 & -0.0560 & -0.0421 & -0.0351 & -0.0480 & -0.0380 & -0.0375 \\
\hline$\zeta_{D}$ & 0.1210 & 0.1231 & 0.1194 & 0.1250 & 0.1241 & 0.1204 & 0.1230 & 0.1211 & 0.1181 \\
\hline$\omega_{n_{D}}$ & 2.2500 & 2.2710 & 2.2310 & 2.2400 & 2.2110 & 2.2010 & 2.2800 & 2.2510 & 2.2290 \\
\hline & & & & & & & & &
\end{tabular}

Table 4. Parameter estimation Accuracy for Lateral-Directional Case

\begin{tabular}{|c|c|c|c|c|c|c|c|c|c|c|}
\hline \multirow{2}{*}{\multicolumn{2}{|c|}{ Parameter }} & \multicolumn{3}{|c|}{ Case I } & \multicolumn{3}{|c|}{ Case II } & \multicolumn{3}{|c|}{ Case III } \\
\hline & & $90 \mathrm{kts}$ & $120 \mathrm{kts}$ & $140 \mathrm{kts}$ & $90 \mathrm{kts}$ & $120 \mathrm{kts}$ & $140 \mathrm{kts}$ & $90 \mathrm{kts}$ & $120 \mathrm{kts}$ & $140 \mathrm{kts}$ \\
\hline \multirow{2}{*}{$C_{Y_{\beta}}$} & Cramer-Rao bound & 0.0143 & 0.0102 & 0.0156 & 0.0131 & 0.0087 & 0.0119 & 0.0076 & 0.0100 & 0.0127 \\
\hline & Standard Deviation & 0.1208 & 0.1103 & 0.0914 & 0.0841 & 0.1146 & 0.1308 & 0.0812 & 0.0633 & 0.0942 \\
\hline \multirow{2}{*}{$C_{l_{\beta}}$} & Cramer-Rao bound & 0.0055 & 0.0062 & 0.0075 & 0.0041 & 0.0043 & 0.0050 & 0.0032 & 0.0093 & 0.0043 \\
\hline & Standard Deviation & 0.0257 & 0.0311 & 0.0214 & 0.0297 & 0.0384 & 0.0311 & 0.0289 & 0.0139 & 0.0215 \\
\hline \multirow{2}{*}{$C_{n_{\beta}}$} & Cramer-Rao bound & 0.0020 & 0.0019 & 0.0037 & 0.0036 & 0.0032 & 0.0032 & - & - & 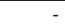 \\
\hline & Standard Deviation & 0.0029 & 0.0013 & 0.0024 & 0.0041 & 0.0028 & 0.0028 & - & - & - \\
\hline \multirow{2}{*}{$C_{Y_{p}}$} & Cramer-Rao bound & 0.0100 & 0.0101 & 0.0120 & 0.0210 & 0.0154 & 0.0121 & 0.0078 & 0.0090 & 0.0124 \\
\hline & Standard Deviation & 0.0061 & 0.0042 & 0.0073 & 0.0033 & 0.0065 & 0.0084 & 0.0073 & 0.0061 & 0.0059 \\
\hline \multirow{2}{*}{$C_{l_{p}}$} & Cramer-Rao bound & 0.0016 & 0.0022 & 0.0032 & 0.0021 & 0.0049 & 0.0013 & - & - & 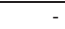 \\
\hline & Standard Deviation & 0.0041 & 0.0011 & 0.0004 & 0.0017 & 0.0031 & 0.0019 & - & - & - \\
\hline \multirow{2}{*}{$C_{n_{p}}$} & Cramer-Rao bound & 0.0087 & 0.0121 & 0.0122 & 0.0096 & 0.0119 & 0.0083 & 0.0129 & 0.0091 & 0.0142 \\
\hline & Standard Deviation & 0.0192 & 0.0144 & 0.0164 & 0.0175 & 0.0143 & 0.0164 & 0.0098 & 0.0084 & 0.0091 \\
\hline \multirow{2}{*}{$C_{Y_{r}}$} & Cramer-Rao bound & 0.0100 & 0.0100 & 0.0100 & 0.0130 & 0.0103 & 0.0055 & 0.0020 & 0.0131 & 0.0100 \\
\hline & Standard Deviation & 0.0082 & 0.0991 & 0.1142 & 0.0122 & 0.1121 & 0.0810 & 0.0911 & 0.0944 & 0.0812 \\
\hline \multirow{2}{*}{$C_{l_{r}}$} & Cramer-Rao bound & 0.0069 & 0.0068 & 0.0000 & 0.0059 & 0.0063 & 0.0020 & 0.0100 & 0.0041 & 0.0096 \\
\hline & Standard Deviation & 0.0482 & 0.0512 & 0.0491 & 0.0421 & 0.0451 & 0.0591 & 0.0551 & 0.0689 & 0.0713 \\
\hline \multirow{2}{*}{$C_{n_{r}}$} & Cramer-Rao bound & 0.0049 & 0.0056 & 0.0050 & 0.0046 & 0.0039 & 0.0050 & - & - & - \\
\hline & Standard Deviation & 0.0012 & 0.0024 & 0.0026 & 0.0049 & 0.0055 & 0.0064 & - & - & - \\
\hline \multirow{2}{*}{$C_{l \delta a}$} & Cramer-Rao bound & 0.0071 & 0.0099 & 0.0099 & 0.0007 & 0.0087 & 0.0100 & 0.0020 & 0.0020 & 0.0087 \\
\hline & Standard Deviation & 0.0300 & 0.0244 & 0.0522 & 0.0412 & 0.0643 & 0.0455 & 0.0573 & 0.0597 & 0.0449 \\
\hline \multirow{2}{*}{$C_{n_{\delta a}}$} & Cramer-Rao bound & 0.0100 & 0.0092 & 0.0099 & 0.0078 & 0.0091 & 0.0099 & 0.0100 & 0.0056 & 0.0009 \\
\hline & Standard Deviation & 0.0012 & 0.0014 & 0.0044 & 0.0063 & 0.0055 & 0.0056 & 0.0106 & 0.0670 & 0.0420 \\
\hline \multirow{2}{*}{$C_{Y_{\delta r}}$} & Cramer-Rao bound & 0.0199 & 0.0129 & 0.0120 & 0.0185 & 0.0110 & 0.0100 & 0.0063 & 0.0070 & 0.0080 \\
\hline & Standard Deviation & 0.1121 & 0.1240 & 0.0890 & 0.0991 & 0.0812 & 0.0957 & 0.0812 & 0.0724 & 0.0931 \\
\hline \multirow{2}{*}{$C_{l_{\delta r}}$} & Cramer-Rao bound & 0.0036 & 0.0032 & 0.0100 & 0.0029 & 0.0000 & 0.0000 & 0.0069 & 0.0059 & 0.0028 \\
\hline & Standard Deviation & 0.0099 & 0.0081 & 0.0074 & 0.0075 & 0.0096 & 0.0105 & 0.0099 & 0.0031 & 0.0063 \\
\hline \multirow{2}{*}{$C_{n_{\delta r}}$} & Cramer-Rao bound & 0.0012 & 0.0021 & 0.0038 & 0.0030 & 0.0028 & 0.0038 & 0.0091 & 0.0085 & 0.0069 \\
\hline & Standard Deviation & 0.0059 & 0.0053 & 0.0421 & 0.0024 & 0.0074 & 0.0085 & 0.0071 & 0.0042 & 0.0053 \\
\hline
\end{tabular}


linear simulation results of longitudinal motion. Mean values of the maximum likelihood estimates were taken to construct a system model for linear simulation. The large difference in the velocity data seems to be caused by the flight test intended for the short period mode which has the characteristic of constant speed.

The linear simulation results of lateral-directional motion are compared with the flight data in Fig. 10. The simulation results show good agreement with the flight data.

The aerodynamic coefficients obtained from the flight data are compared in Table 5 and Table 6 with those from the wind tunnel test and analytical methods such as CFD and DATCOM.

The lift coefficient slope, $C_{L_{a}}$ obtained from the flight data agrees with the wind tunnel test data, while DATCOM and the CFD results are slightly larger. It is conjectured that the analytical methods such as DATCOM and CFD do not accurately estimate the downwash on the main wing due to the canard.

The longitudinal static stability parameter, $C_{M_{\alpha^{\prime}}}$ computed from the estimated neutral point coincides well with the power off wind tunnel prediction. The parameter estimation (MLE) output of the flight data for $C_{M_{\alpha}}$ is slightly less than the wind tunnel test data. Considering that the wind tunnel test was performed for power-off condition, this excellent agreement between the flight test results and the wind tunnel test results does not mean these data are accurate. The power effect on $C_{M_{\alpha}}$ is neither large nor negligible. The magnitude of $C_{M_{a}}$ computed from DATCOM is too small and the CFD output is large compared with the flight test results.

On the whole, for the longitudinal case, the results from the MLE of the flight data almost coincide with those from

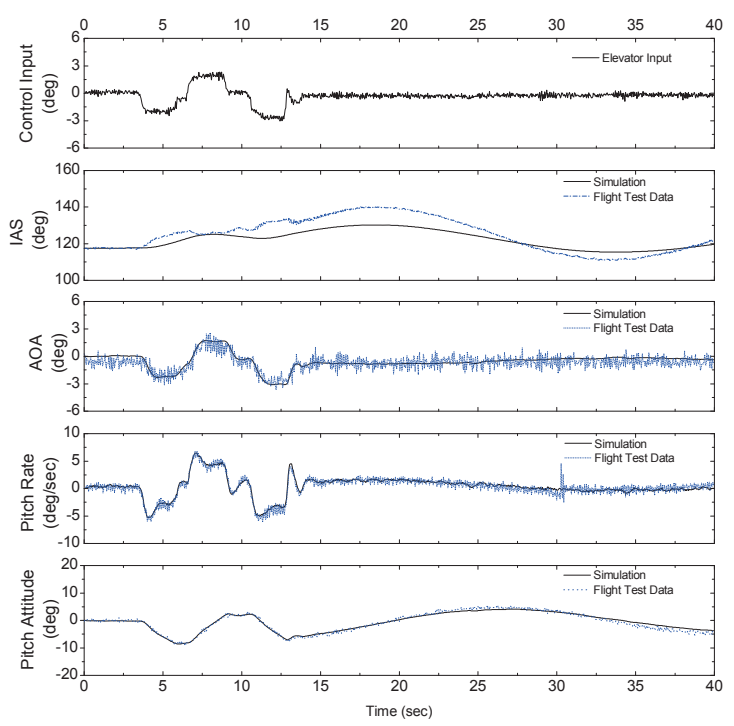

Fig. 9. Comparison of the Flight Data and Linear Simulation Results for Longitudinal Case

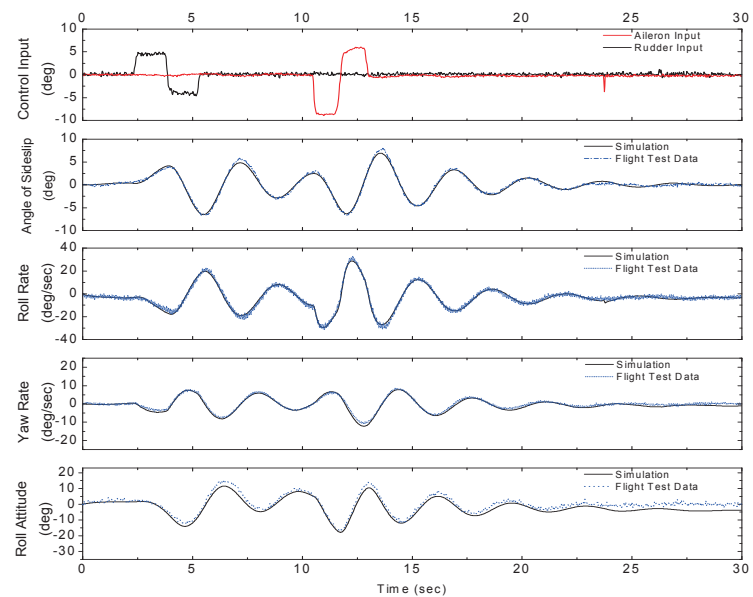

Fig. 10. Comparison of the Flight Data and Linear Simulation Results for Lateral-Directional Case 
the wind tunnel test results, except for $C_{D_{\alpha}}$ and $C_{D_{\delta e}}$. These aerodynamic derivatives related to drag are difficult to estimate from the flight data since their sensitivities to the measurement data in the short period mode are not large enough.

Table 5 and Table 6 also show that the DATCOM output is very unreliable for the canard aircraft. The CFD output is also not accurate. Therefore we must be very careful when we use the analytical results during a preliminary design phase of canard aircraft.

The longitudinal aerodynamic derivatives of the Firefly and the Velocity are close to each other. This result was expected since the Firefly was designed by modifying the Velocity with the intention of improving the lateral-directional dynamic flying qualities only. Therefore, it is reasonable that the short period damping and natural frequency of the Firefly are close to those of the Velocity. The Phugoid damping and the natural frequency of the Firefly and the Velocity differ somewhat. This difference is probably caused by the poor estimation of the parameters that mainly influence the Phugoid mode.

Table 6 indicates that the steady state results obtained by solving the equilibrium equations are generally close to the MLE output, except for $C_{l_{\beta}}$. The flight determined derivatives agree well with the wind tunnel test results, except for the derivatives $C_{n_{\beta}}, C_{l \beta}$, and $C_{n_{\delta e}}$

The magnitude of $C_{l_{\beta}}$ obtained from the MLE method is larger than the wind tunnel result and the steady state flight test result. The directional stability derivative, $C_{n_{\beta}}$, obtained from the flight data is smaller than the wind tunnel test result and the results from the analytical method of DATCOM and AAA.

The magnitude of the derivative, $C_{n_{\delta \alpha}}$ obtained from the flight data is also smaller than the wind tunnel test result and the results from the analytical method. Since the magnitude of $C_{n_{\delta a}}$ that determines the adverse yaw of the aircraft is very

Table 5. Comparison of Results for Longitudinal Case

\begin{tabular}{|c|c|c|c|c|c|c|c|c|c|}
\hline Parameter & DATCOM & $\overline{\mathrm{AAA}}$ & CFD & $\begin{array}{r}\text { Wind } \\
\text { Tunnel }\end{array}$ & $\mathrm{R} / \mathrm{C}$ Test & $\begin{array}{r}\text { Steady } \\
\text { State } \\
\end{array}$ & MLE & Velocity & $\begin{array}{r}\text { Cessna } \\
182 \\
\end{array}$ \\
\hline$C_{D_{\alpha}}$ & 0.1150 & 0.2050 & - & 0.3200 & - & - & 0.5805 & 0.6030 & 0.1210 \\
\hline$C_{L_{\alpha}}$ & 6.5000 & 6.3400 & 6.5500 & 5.5000 & 5.3300 & 5.7000 & 5.5669 & 5.2100 & 4.4100 \\
\hline$C_{M_{\dot{\alpha}}}$ & - & 0.5500 & - & - & - & - & 2.3172 & 2.3900 & -7.2700 \\
\hline$C_{M_{\alpha}}$ & 0.2900 & 0.8800 & 0.7450 & 0.6300 & 0.5690 & 0.6250 & 0.5968 & 0.5910 & -0.6130 \\
\hline$C_{L_{q}}$ & 1.8570 & 5.9600 & - & - & - & - & - & - & 3.9000 \\
\hline$C_{m_{q}}$ & 6.5400 & 7.4900 & - & - & - & - & 13.853 & 14.010 & -12.400 \\
\hline$C_{D_{\delta e}}$ & 0.0167 & 0.0104 & - & 0.0160 & - & - & 0.0001 & 0.0020 & 0.0000 \\
\hline$C_{L_{\delta e}}$ & 0.3900 & 0.3140 & - & 0.3000 & 0.5620 & - & 0.2543 & 0.2890 & 0.4300 \\
\hline$C_{m_{\delta e}}$ & 0.5530 & 0.550 & - & 0.7400 & 1.7370 & - & 0.7759 & 0.7010 & -1.1220 \\
\hline$\zeta_{s p}$ & - & 0.4220 & - & - & - & - & 0.6273 & 0.7030 & 0.74420 \\
\hline$\omega_{n_{s p}}$ & - & 4.5300 & - & - & - & - & 4.2020 & 4.2900 & 4.9802 \\
\hline$\zeta_{p h}$ & - & 0.0513 & - & - & - & - & 0.0549 & 0.0943 & 0.1189 \\
\hline$\omega_{n_{p h}}$ & - & 0.1460 & - & - & - & - & 0.1371 & 0.2030 & 0.2311 \\
\hline
\end{tabular}

Table 6. Comparison of Results for Lateral-Directional Case

\begin{tabular}{cccrrrrrrr}
\hline Parameter & DATCOM & AAA & CFD & $\begin{array}{r}\text { Wind } \\
\text { Tunnel }\end{array}$ & R/C Test & $\begin{array}{r}\text { Steady } \\
\text { State }\end{array}$ & MLE & Velocity & $\begin{array}{r}\text { Cessna } \\
182\end{array}$ \\
\hline$C_{Y_{\beta}}$ & 0.5620 & 0.4280 & - & 0.4480 & - & 0.5380 & 0.5210 & 0.6130 & 0.3930 \\
\hline$C_{l_{\beta}}$ & 0.0680 & 0.0460 & - & 0.0500 & - & 0.0590 & 0.0740 & 0.0930 & 0.0923 \\
\hline$C_{n_{\beta}}$ & 0.0600 & 0.0480 & - & 0.0500 & - & 0.0310 & 0.0350 & 0.0240 & 0.0587 \\
\hline$C_{Y_{p}}$ & 0.0640 & 0.0021 & - & - & - & - & 0.0021 & 0.0390 & 0.0750 \\
\hline$C_{l_{p}}$ & 0.5020 & 0.6230 & - & - & - & 0.4930 & 0.4600 & 0.4710 & 0.4840 \\
\hline$C_{n_{p}}$ & 0.0272 & 0.0244 & - & - & - & - & 0.0520 & 0.0590 & 0.0278 \\
\hline$C_{Y_{r}}$ & - & 0.2011 & - & - & - & - & 0.2130 & 0.1810 & 0.2140 \\
\hline$C_{l_{r}}$ & 0.0812 & 0.0965 & - & - & - & - & 0.0970 & 0.1130 & 0.0798 \\
\hline$C_{n_{r}}$ & 0.0550 & 0.0572 & - & - & - & - & 0.0700 & 0.0570 & 0.0937 \\
\hline$C_{l_{\delta a}}$ & 0.1630 & 0.1220 & - & 0.1050 & - & 0.1120 & 0.1250 & 0.0920 & 0.2290 \\
\hline$C_{n_{\delta a}}$ & 0.0033 & 0.0046 & - & 0.0032 & - & - & 0.0025 & 0.0100 & 0.0216 \\
\hline$C_{Y_{\delta r}}$ & 0.2310 & 0.2560 & - & 0.1650 & - & 0.1110 & 0.1490 & 0.0912 & 0.1870 \\
\hline$C_{l_{\delta r}}$ & 0.0115 & 0.0114 & - & 0.0100 & - & 0.0090 & 0.0110 & 0.0080 & 0.0147 \\
\hline$C_{n_{\delta r}}$ & 0.0620 & 0.0770 & - & 0.0450 & - & 0.0350 & 0.0410 & -0.024 & 0.0645 \\
\hline$\zeta_{D}$ & - & 0.1412 & - & - & - & - & 0.1231 & 0.1092 & 0.1556 \\
\hline$\omega_{n_{D}}$ & - & 2.3419 & - & - & - & - & 2.2710 & 1.9134 & 3.1248 \\
\hline
\end{tabular}


small compared with other parameters, $C_{n_{\delta a}}$ is difficult to estimate accurately from the flight data. The derivative, $C_{n_{\delta a}}$, predicted by the analytical method, is generally not reliable.

The aileron control effectiveness derivative, $C_{l \delta d_{d}}$, estimated from the flight data, agrees with other results, except for the DATCOM output which is too large.

The rudder control derivatives estimated from the MLE method coincide with the wind tunnel test results while they are slightly larger than the steady state flight test output and smaller than the analytical results.

The roll damping derivative, $C_{l p}$, estimated from the flight data, is close to the DATCOM output. The derivatives, $C_{Y_{p}}$ and $C_{n_{p}}$, do not agree with the analytical results. These disagreements are anticipated since the Cramer-Rao bounds of these derivatives are large.

The yaw damping derivative, $C_{n_{r}}$, estimated by the MLE method is larger than the analytical results.

Table 6 also shows that the Dutch roll damping of the Firefly is larger than that of the Velocity. This means that the goal of the development of the Firefly is satisfied since the Firefly was designed with the intention of improving the lateral-directional flying qualities by locating the vertical tails afterward and by decreasing the main wing sweepback angle.

Cessna-182 data is listed in Table 5 and 6 for the comparison of conventional aircraft data with the canard aircraft data. It is noticeable that the signs of $C_{M_{\delta e}}$ and $C_{M_{\alpha}}$ of the Firefly are opposite to those of the Cessna-182. This is due the difference of horizontal tail location with respect to the main wing.

\section{Concluding Remarks}

The longitudinal and lateral-directional aerodynamic derivatives for the Firefly, a four seat general aviation canard aircraft, were estimated from the flight data by using a maximum likelihood estimation method, solving steady state trim equations, and comparing with the results from a wind tunnel test and analytical prediction methods such as DATCOM, AAA, and CFD. Even though the prediction of aerodynamic derivatives of the canard aircraft by analysis or wind tunnel test is difficult, the parameter estimation of the canard aircraft does not differ from that of conventional aircraft.

For the lateral-directional derivatives, the flight determined derivatives agree well with the wind tunnel test data, except for the derivatives, $C_{n_{\beta}}, C_{l_{\beta}}$, and $C_{n_{\delta a}}$.

For the longitudinal derivatives, on the whole, the results from the flight data agree well with the wind tunnel test results except the aerodynamic derivatives related to drag. The analytical results from DATCOM, AAA, and CFD are shown to be poor. Therefore, it is strongly recommended that new aerodynamic empirical database should be constructed for canard aircraft.

In this study, comparisons of the flight determined parameters with the wind tunnel test results and the results from the analytical methods were made only for one flight condition, that is, for one angle of attack. For further study, more extensive flight tests need to be performed so that the parameter variations for the various angles of attack could be investigated and analyzed.

\section{References}

[1] Daniel P. Raymer, Aircraft Design: A Conceptual Approach, AIAA Education Series, 2000

[2] E. T. Kim, et al., "A study on a Configuration Design of the Firefly", Proceedings of the KSAS Fall Annual Meeting, 2002.

[3] S. M. Ahn, et al., "A study on a Subsystem Design of the Firefly", Proceedings of the KSAS Fall Annual Meeting, 2002.

[4] E. T. Kim and J. D. Chung, "Wind Tunnel Investigation of Aerodynamic Characteristics of a Small Canard Aircraft", Journal of the Korean Society for Aeronautical and Space Sciences, Vol. 29, No. 7, pp.1-8, October 1, 2001.

[5] Chung Jindeong, Cho Tahwan, Lee Jangyeon and Sung Bongzoo, "Wind Tunnel Test of a Canard Airplane", KSME International Journal, Vol. 16, No.1, pp. 125-131, 2002.

[6] S. W. Choi and E. T. Kim, "Computational Flow Analysis around Canard Aircraft", Proceedings of the KSAS Spring Annual Meeting, pp.121-124, 1999.

[7] S. W. Nho, et al., "Parameter Estimation of Aerodynamic Stability Derivatives of Canard Type Aircraft Using Extended Kalman Filter", Proceedings of the KSAS Spring Annual Meeting, 2001.

[8] D. W. Jeong, et al., "Parameter Estimation of Aerodynamic Controllability/Stability Derivatives of Canard Type Aircraft by Flight Test", Proceedings of the KSAS Spring Annual Meeting, 2001.

[9] M. Y. Hwang, et al., "Lateral Stability/Control Derivatives Estimation of Canard Type Airplane from Flight Test", Proceedings of the ICCAS, 2001.

[10] Anon, USAF Stability and Control DATCOM, U.S. Air Force Flight Dynamics Lab, Wright-Patterson Air Force Base, Dayton, 1974.

[11] Jan Roskam, Airplane Design: Part I - Part VII, Roskam Aviation and Engineering Corporation, USA, 1985,

[12] Eugene A. Morelli, "Flight Test Validation of Optimal 
Input Design and Comparison to Conventional Inputs", AIAA Atmospheric Flight Mechanics Conference and Exhibit, 1997.

[13] Introduction to Performance and Flying Qualities Flight Testing, National Test Pilot School, 2000

[14] Donald T. Ward, Introduction to Flight Test Engineering, Elsevier Science Publishers, 1993.

[15] Iliff, K.W., Maine, R.E., Practical Aspects of Using a Maximum Likelihood Estimation Method to Extract Stability and Control Derivatives from Flight Data, NASA TN D-8209, 1976

[16] Maine, R.E., Iliff, K.W., Identification of Dynamic Systems: Theory and Formulation, NASA RF 1168, June 1986.

[17] Ljung, L. System Identification: Theory for the User,
Prentice Hall, Englewood Cliffs, NJ, 1987

[18] Ravindra Jategaonkar, et al., Maximum Likelihood Estimation of Parameters in Linear Systems with Process and Measurement Noise, DFVLR-FB 87-20

[19] E. T. Kim, et al., "A Study on a Canard Aircraft Flight Characteristics through Flight Test", Proceedings of the KSAS Spring Annual Meeting, 2000.

[20] K. J. Seong, et al, "An Analysis of Longitudinal Trim Condition for the Firefly", Proceedings of the KSAS Fall Annual Meeting, 2002.

[21] S. J. Kang, et al, "A Study on Estimation of LateralDirectional Aerodynamic Derivatives of a Small Canard Aircraft", Proceedings of the KSAS Fall Annual Meeting, 2002. 